\title{
Challenging the Evolutionary Strategy for Synthesis of Analogue Computational Circuits
}

\author{
Yerbol A. Sapargaliyev, Tatiana G. Kalganova
}

Brunel University, London, UK.

Email: \{yerbol.sapar, Tatiana.kalganova\}@brunel.ac.uk

Received July 21 $1^{\text {st }}, 2010$; revised August $27^{\text {th }}, 2010$; accepted September $2^{\text {nd }}, 2010$.

\begin{abstract}
There are very few reports in the past on applications of Evolutionary Strategy (ES) towards the synthesis of analogue circuits. Moreover, even fewer reports are on the synthesis of computational circuits. Last fact is mainly due to the difficulty in designing of the complex nonlinear functions that these circuits perform. In this paper, the evolving power of the ES is challenged to design four computational circuits: cube root, cubing, square root and squaring functions. The synthesis succeeded due to the usage of oscillating length genotype strategy and the substructure reuse. The approach is characterized by its simplicity and represents one of the first attempts of application of ES towards the synthesis of "QR" circuits. The obtained experimental results significantly exceed the results published before in terms of the circuit quality, economy in components and computing resources utilized, revealing the great potential of the technique proposed to design large scale analog circuits.
\end{abstract}

Keywords: Analog Circuit Synthesis, Evolutionary Electronics, Computational Circuits, SPICE

\section{Introduction}

The Evolvable Hardware (EHW) is one of the most promising areas of today's electronics. The EHW where the ultimate goal is an electronic circuit is also known as Evolutionary Electronics (EE) [1]. Evolutionary Algorithms (EA) together with a circuit simulation tool (or real hardware) automatically designs the circuit for a given problem. This approach uses very little knowledge of conventional circuit design theory and is mainly based on the exploitation of "search and test approach".

In general, EA navigated by fitness values, provides randomly created and mutated chromosomes. Each chromosome encodes a structure for a circuit in a form of genotype and has to be evaluated by a fitness function. The fitness function assigns each chromosome with a fitness value that defines how close the current hardware structure is to the target by its functioning. The circuits evolved may have unconventional designs and less of all depend on the personal knowledge of a designer. Nowadays, the EA is represented by Genetic Algorithm (GA), Genetic Programming (GP) and ES. While GA is definitely the most popular tool, GP is rapidly developing in recent years and is notable by its outstanding results in the area of EE. On the other hand, ES that first was introduced in [2], can be named as the simplest EA due to it does not use the crossover between chromosomes during mutation stage; each mutation involves only one chromosome. One of the main targets of this paper is to discover the potentials of ES in evolving large analog circuits. The second column of Table 1 carries the statistics on types of EAs used in other works.

The analogue CCs play an important role in applications where it is required to have a limited number of mathematical functions. They suggest an economy in components eliminating the analogue-digital-analogue conversion that conventional designs usually incorporate, and provide considerably shorter delay in circuit response. The vitality of CCs is well described in [3].

Today the open-ended methods of evolutionary analog circuit synthesis are questioned (i.e., in $[1,4]$ ) with an important issue, whether they are able to create solutions that are valid and trustworthy enough being realized in silicon? In [5] the set of experiments have proven that the open-ended methods enabled to design low/high-pass filters with topology-based robustness. In [6] the frequency discriminator robust to wide temperature range was evolved with an open-ended GA intrinsically in FPGA. The [7] describes experiments that allowed adaptive in-situ circuit reconfiguration in extreme temperature and radiation environments. In [8], the unconstrained evolution successfully created the analog vari- 
ability-tolerant CMOS circuits performing XOR and XNOR functions. The literature review on that subject enables to distinguish two approaches. The first traditional one follows the paradigm wherein the evolution is, firstly, set to discover the unconventional design and later the circuit is tuned to improve the robustness [6,7,9-11]. Another approach suggests for use the evolutionary system that originally purposed for the robust designs $[4,5,8]$. In a current work, we go along the first way focusing on exploration of technique's capabilities to create unconventional designs, leaving the evolution of robustness for the next stage experiments.

The next section overviews the previous work in the area. Section 3 introduces the whole evolutionary technique. Section 4 describes the experimental results together with comparison with the results obtained before. And, finally, the last section concludes the paper.

\section{Previous Work}

In the past the low-pass filters [5,10-16], high-pass filters $[5,10,11,13,17,18]$ and amplifiers $[1,9,11,12]$ were successfully synthesized with the help of EA. In [1] the unconstrained evolution, both spatially and temporally had been applied towards a digital reconfigurable hardware FPGA. By releasing the full repertoire of behavior that FPGA can be manifest, namely, allowing any connections among modules, letting to evolve the granularity and synchronization, evolution had been able to find a highly efficient electronic structure, which requires 1-2 orders less silicon area to achieve the same performance as conventional design does. Natural behavior of analogue components started to be exploited inside a digital circuit.

In analogy to this approach, the unconstrained evolution in our previous endeavor to sharpen our technique was applied in [16] towards the originally analogue circuits (low-pass filters) and excellent results were retained. Most of the works in the area start from evolving a low-pass filter (Table 1). The last one is a convenient tool for the probation of evolutionary technique and tuning the EA parameters towards the more sophisticated designs. In this paper, we tried to evolve the computational circuits (CCs) that perform the: cube root, cubing, square root [19] and squiring functions. CCs, in contrast to filters, enable a comparison of resulted designs with circuits published before much easier. This is due to fewer numbers of characteristics that describe the circuit. A filter, besides main characteristics such as a stop-, pass- and transition-bands, has the attenuation values and ripples, which are difficult to count during the comparison. In contrast, a CC is characterized only by average error of the computing function, which together with circuit size (component amount) and evaluation efforts gives the
Table 1. Advances on the evolution of analogue circuits.

\begin{tabular}{lccc}
\hline \multicolumn{1}{c}{ Researcher } & EA type & GLVS & CSCR \\
\hline Koza et al. [9] & GP & ILG & Partially \\
Mydlowec et al. [3] & GP & ILG & Partially \\
Streeter et al. [20] & GP & ILG & Partially \\
Lohn, Colombano [11] & GA & ILG & Yes \\
Goh, Li [12] & GA & ILG & Yes \\
Zebulum, et al. [10] & GP,GA & ILG,OLG,UDIP & Yes \\
Grimbleby [14] & GA & ILG & Data n/a \\
Dastidar, et al. [21] & GA & OLG & Yes \\
Ando, Iba [13] & GP,GA & Data n/a & Yes \\
Sripramong et al. [22] & GP & Fixed & Yes \\
Walker et al. [8] & GA & Fixed & Partially \\
Chang, Hou, Su [18] & GP & UDIP & Yes \\
Mattiussi, Floreano [23] & GA & OLG & Yes \\
Gan, Yang et al. [17] & CS & OLG & Yes \\
McConaghy et al. [4] & GP & ILG & Yes \\
Kim et al. [5] & ES & ILG & Data n/a \\
Walker et al. [8] & GP + ES & OLG & No \\
Das A., Vemuri [24] & GA & UDIP & Yes \\
Sapargaliy- LCR [16] & OLG & ES & No \\
ev et al. $\quad$ QR(current) & OLG & ES & No \\
\hline
\end{tabular}

CSCR is for circuit-structure-checking rules; PO is for parameter optimization; GLVS is for the genotype length varying strategies; CS is for Clonal Selection.

sufficient picture to judge on capabilities of the methodology.

Works [10,13] gave the comparison between GP and GA. The first work was made as an analogy to the biology concept with comparison of different types of variable length genotypes strategies, whereas in the second one it was intrinsic evolution of a real hardware for robustness purposes. According to [10], the "genotype length varying strategies" refer to the way in which the chromosome's lengths are sampled by the EA at each generation. It is easy to follow this idea if one looks at sizes of the best circuits throughout the generations. There were different kinds of strategies introduced in [10], where two of them have shown excellent results: Increasing Length Genotypes (ILG) and Oscillating Length Genotypes (OLG). If it occurred that the size of the best circuit at each following generation never decrease than it is ILG, otherwise it is OLG. The OLG strategy is a kind of ILG in which the genotypes are also allowed to decrease in size. The main purpose of OLG is to create pathways from large to smaller genotypes with improved fitness values. The third column of Table 1 summarizes the information on OLG vs. ILG.

Most of the works focus on such circuits like filters and amplifiers which, we think, are not an adequate enough challenge for the probation of the up-to-date evolutionary 
techniques. Current research is devoted to CCs which are the ones of most provoking issues for any automatic circuit synthesis system. It should be mentioned that the largest analog circuit evolved in the area of EE is a squire root circuit with 64 components in [9]. We found another three papers on CCs $[3,24,25]$ that regarded the same circuits as in this paper. In $[3,9,20]$ they used GP circuit-constructing program trees approach with four kinds of functions. They also used automatically defined functions and potentially enabled certain substructures to be reused. The paper [9] suggests an attractive opportunity among all others for judging on effectiveness of the evolutionary tool. Targeting to the same arithmetic functions and utilizing an identical evaluation procedure (fitness function), one can directly compare the fitness values (average error), circuit size (economy) and PC time spent. In this paper, we took advantage of this opportunity.

In [3] two CCs were developed by the similar evolutionary technique as in [9], however they used time-continues signals in time-domain simulations. The transient analysis of a circuit in contrast to DC-analysis provided more robust circuits despite the higher time-consumption to complete the analysis. The patent in [25] presents the conventionally designed cubing $\mathrm{CC}$, that was improved in [19] by iterative refinement method. Both are taken for comparison in Section 4.

The work in this paper contributes to the following issues of EE: a) discovers the potentials of ES towards the design of nonlinear analog circuits, and b) reveals the ability of unconstrained evolution to find more efficient and unconventional designs.

\section{Evolutionary Technique of QR Circuits}

Reaching successful circuits most of all depends on an evolutionary technique that worked out and applied. The last one is a set of rules according to which, parameters of EA (e.g., mutation rate, crossover, selection, etc.), genotype length varying strategies [10], mutation types and the circuit representation technique are managed.

\subsection{Encoding (Representation)}

We use only three types of components as in [3,9]: Qn the n-p-n bipolar transistor, Qp - the p-n-p bipolar transistor and $\mathrm{R}$ - resistor. The linear circuit representation proposed for use, i.e., every component of a circuit represented as a particular gene, and each gene consisted

\section{$\mathrm{Rx} \quad \mathrm{N} 1 \quad \mathrm{~N} 2 \mathrm{~Pa}$}

(a)
QX N1 N2 N3

(b)
Figure 1. A gene coding (a) resistor; (b) bipolar transistor. Rx-loci and Pa-loci are the resistor's name and parameter; Qx-loci is the transistor's name; N1, N2, N3-loci are the nodes for the first, the second and the third pins. of 4 loci corresponding to component's features: name, node numbers to each pin and parameter (only for R). On Figure 1 is a view of a gene coding a resistor (a) and a bipolar transistor (b). The gene looks exactly the same as a component line in the PSPICE netlist, so, there is no necessity to convert a genotype into a netlist. The simplicity of linear representation we utilized simplifies the terminology, for example, we mean "a circuit" when we mention "a chromosome", we mean "a component" when we mention "a gene", we mean "a population" when we mention "a netlist", and vice versa.

For a resistor's Pa-loci, we set 64 possible values of $\mathrm{E}-12$ series, i.e., there were 5 decades from $10 \Omega$ to $1 \mathrm{E}+6$ $\Omega$ available for evolution, plus four additional parameters.

\subsection{Unconstrained Evolution of “QR” Circuit}

In an analogue domain, the circuit-structure-checking rules at the netlist composition stage that prohibit some circuits to be tested/simulated were regarded as the main constraints in evolution. The target of these rules is usually increasing the portion of rightly analyzed circuits as well as the avoiding time consumptions during resolving the implausible designs by simulation software. In [16] we called "absolutely unconstrained evolution of an analogue circuit" the process of circuit netlist generation during which no circuit-structure-checking rules applied and all the circuits are counted as valid graphs except ones that have components with dangling nodes and with isolated sub-circuits. We utilized special technique that enabled us to avoid most of the errors inherent to circuits built up of reactive components inductors and capacitors.

In a current work we use Qn, Qp and R components. Here transistors can lead to unconvergences during analysis. Therefore, in most of the works in Table $\mathbf{1}$ (column 4) the rules banning some transistor connections, such like emitter-to-collector and base-to-PS, were applied. In this paper, we did not apply any prohibitions to any kind of connections. We also did not prohibit the formation of "loops" of components during circuit growth. "Loop" (or sub-circuit) is a component or a group of components aside from the main circuit that does not connect to the main circuit or connects to it only via alone node. "Loops" mainly do not influence on functionality of the whole circuit, however, they participate in carrying the neutral mutations [1]. The statistics on constraint/unconstraint methods in the area of analog EE are shown in the fourth column of Table 1.

The transient analysis applied to perform the evaluation instead of DC-analysis. Due to the tolerance that transient analysis expresses to the same circuits that under the DC-analysis are treated as unconvergent [26], we could maximize the portion of valid chromosomes in each population. This hint allows the multitude of indi- 
viduals that could potentially carry the right structures, to pass on to the next generation, making significant contribution to the unconstraint evolution.

\subsection{Experiment Settings}

The embryo circuit is the component or a number of components (including the voltage source), that can be predetermined for the particular targeted circuit to ease the further circuit growth. We defined the embryo circuit for all four kinds of our targets the same: a pulse voltage source, source resistance Rsource $=1 \mathrm{k} \Omega$ and the load resistance $\mathrm{Rload}=1 \mathrm{k} \Omega$. These three components on Figure 2 compose the embryonic circuit and are absolutely identical to that ones in most of the works in Table 1. The embryo also has two sources of direct voltage suggesting the evolution to choose between (or use both) $+15 \mathrm{~V}$ and $-15 \mathrm{~V}$, so that the initial node number at a start is five.

Figure 3 generally shows the algorithm of the experiment. It consists of 4 main blocks. The PC program written in $\mathrm{C}$ programming language described all four parts and unites them in one code.

The Start-block provides population of chromosomes in the form of PSPICE netlist. This block includes all the data necessary for embryo circuit production. Being delivered to ES block, every chromosome at this stage is grown up from the embryo to the individuals with the same number of genes. At first generation to be analyzed all chromosomes consisted of three components.

ES block performs the particular procedures of ES, such as: cloning them the best chromosomes, mutating and checking for termination criteria. It modifies the genotype and produces the population of chromosomes in

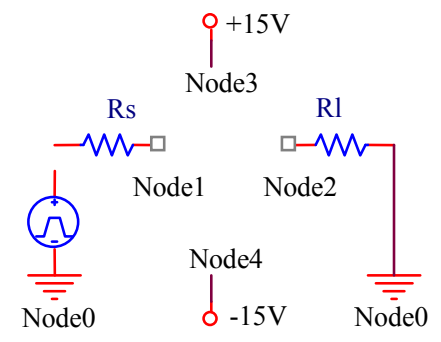

Figure 2. Embryo circuit.

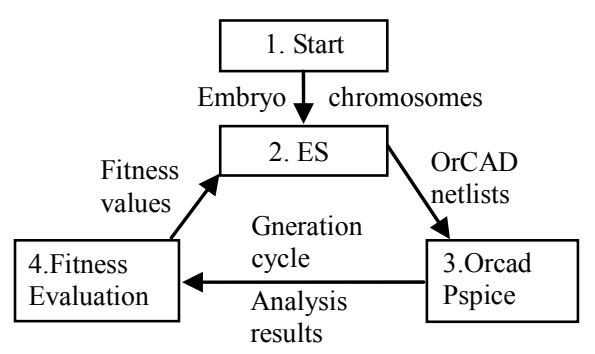

Figure 3. Experimental system. a form of cir-batch-file towards the PSPICE. Last one is utilized in non-interactive batch simulation mode.

Block 3 runs PSPICE, downloads cir-file and receives the result from PSPICE in a form of out-file, then passes it for evaluation to Block 4 . Block 4 contains the fitness function that evaluates and assigns each chromosome with a fitness value.

The mutation process is applied to each chromosome except the chromosome with the best fitness value. It stays as reference for others during the generations. Depending on correlations between the best and current chromosome's lengths/fitness each individual is put under one of the particular mutations.

Add_new_component_mutation (ANEM) and Delete_component_mutation (DEM) are the procedures, during which one randomly generated gene is added/removed to/from each chromosome. ANEM or DEM applies depending on whether is the genotype length of current chromosome shorter/longer of that one of the best chromosome's. Due to DEM the circuits evolved are supposed to be modest in components.

The Circuit_structure_mutation (CSM) performs mutation over every loci of randomly chosen gene including the component name, parameter or pin connections, without changing the chromosome length. In CSM, despite the total amount of components stays unchangeable, the number of circuit nodes could be reduced or increased.

\subsection{Substructure Reuse}

The modification of chromosome by junction it with a substructure is a kind of mutation routine that brings, however, a radical modification. If an ordinary mutation brings $3-5 \%$ of a new genotype, the substructure reuse mutation (SUM) is applied regardless a mutation rate and can exceed $50 \%$. For instance, in the case of CCs in this paper we, firstly, tried to reach the targets without SUM, based only on three mutations mentioned in previous subsection. The best fitness that seemed to be rapidly improving at a start, later, when genotype length reached 12-17 genes, slowly and irrevocably decayed. And only the introduction to a system the SUM, could make the experiment progress. The system, automatically utilizing the best chromosomes of miner sizes (limited from 2 to 7 genes) collected at earlier generations and joining them to stagnated chromosomes, was able to bring up to $60 \%$ of new genotypes in our experiments.

It should be noticed that the SUM procedure is purposed to be used only toward chromosomes that showed the signs in a fitness decline during the previous generations. This approach differs from that one where the substructures accumulated in a permanent database are used intensively along with single components [4,21]. The wide use of substructures that already are silicon-proven 
could fasten the road of evolved designs to a commercial application, but in the scope of this paper, as mentioned in Section 1, we focus first on the exploring power of the evolutionary system.

Altogether ANEM-CSM-DEM-SUM enables to hold a population within restrained chromosome's lengths and for evolution to be more focused. In general, each chromosome in a population is allowed gradually grow, where the speed of growth is controllable with the help of an automatic/manual tunable threshold coefficient.

\subsection{Fitness Function}

The target for evolutionary search is to evolve four CCs which output voltages are: the cube root, cube, square root and square of their input voltages. To enable ourselves to make the estimation of the final results, we have set the same fitness terms as in [9] for all four cases. That are, we made the PSPICE simulator to perform a transient analysis of a source signal of length 0.2 second at 21 equidistant time-points; the voltage source forms a pulse signal arising from $-250 \mathrm{mV}$ to $+250 \mathrm{mV}$ for the cube root, cubing and squaring, and from $0 \mathrm{mV}$ to +500 $\mathrm{mV}$ for the square root [19]. A fitness value is set to the sum, over these 21 fitness cases of the absolute weighted deviation between the target value and the actual output value: $F=\sum_{i=0}^{p}\left|V_{\text {ideal }}^{i}-V_{\text {measured }}^{i}\right|$, where $V_{\text {ideal }}^{i}$ is the voltage in $i$-th point for the ideal response and $V_{\text {measured }}^{i}$ is the voltage in the i-th point obtained for the evolved circuit; $p$ is a number of points evaluated equaling 21 . The smaller the fitness value, the closer the circuit is to the target; the fitness penalizes the output voltage by 10 if it is not within $1 \%$ of the target voltage value.

The circuits that were treated by PSPICE as error carrying were assigned to the worst fitness. We set as a termination criterion reaching either the fitness value did not improve over 20 consecutive generations or the best circuit exceeded 70 components in size.

\section{Experimental Results}

The results presented are out of 5 runs for each case with different seeds for the random number generator. The aggregated data for all 20 runs are presented in Table 2, where the best runs are marked in bold. We used 10 PCs with Pentium-4/2.8 GHz processor running at the same time independently from each other. The average time per experiment is 43 hours. The total population was 30000 individuals, mutation rate $5 \%$ and selection $10 \%$.

The best-of-run circuit (Figure 4) for the problem of designing a square root circuit has 23 components with the fitness 0.194. The best-of-run circuit (Figure 5) for the problem of designing a squiring circuit has 35 com-
Table 2. Statistics for evolution of the $\mathbf{4}$ targeted circuits.

\begin{tabular}{ccccccc}
\hline \multirow{2}{*}{ No } & Fitness & $\begin{array}{c}\text { Com- } \\
\text { ponent } \\
\text { No }\end{array}$ & $\begin{array}{c}\text { Gen- } \\
\text { eration } \\
\text { No }\end{array}$ & Fitness & $\begin{array}{c}\text { Compo- } \\
\text { nent No }\end{array}$ & $\begin{array}{c}\text { Gen- } \\
\text { eration } \\
\text { No }\end{array}$ \\
\cline { 2 - 7 } & \multicolumn{3}{c}{ Square Root } \\
\hline 1 & 0.283 & 43 & 119 & $\mathbf{0 . 0 3 0 2}$ & Squaring \\
2 & $\mathbf{0 . 1 9 4}$ & $\mathbf{2 3}$ & $\mathbf{1 2 3}$ & 0.0459 & 43 & $\mathbf{9 2}$ \\
3 & 0.443 & 50 & 208 & 0.0563 & 48 & 143 \\
4 & 0.798 & 38 & 97 & 0.0951 & 38 & 97 \\
5 & 0.255 & 50 & 200 & 0.0776 & 50 & 135 \\
\hline \multicolumn{7}{c}{ Cube Root } \\
1 & 0.764 & 44 & 115 & 0.0095 & 50 & 195 \\
2 & 1.060 & 49 & 179 & 0.0205 & 38 & 72 \\
3 & $\mathbf{0 . 2 5 1}$ & $\mathbf{3 9}$ & $\mathbf{1 5 2}$ & 0.0079 & 49 & 109 \\
4 & 0.268 & 50 & 201 & $\mathbf{0 . 0 0 6 1}$ & $\mathbf{4 4}$ & $\mathbf{7 8}$ \\
5 & 0.643 & 40 & 294 & 0.0101 & 37 & 98 \\
\hline
\end{tabular}

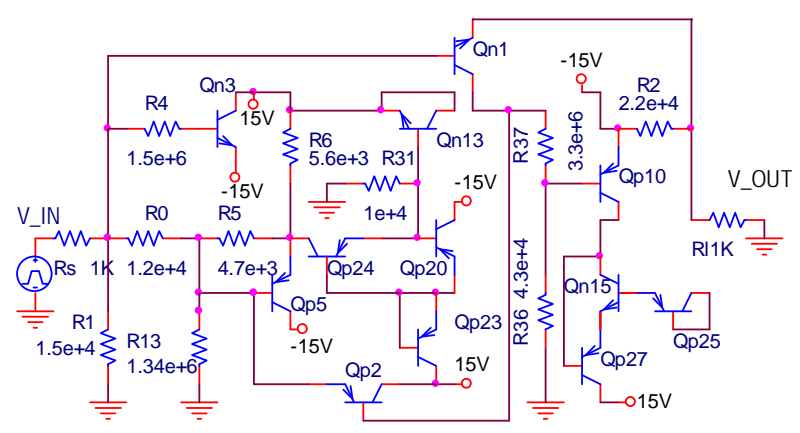

Figure 4. The evolved square root circuit.

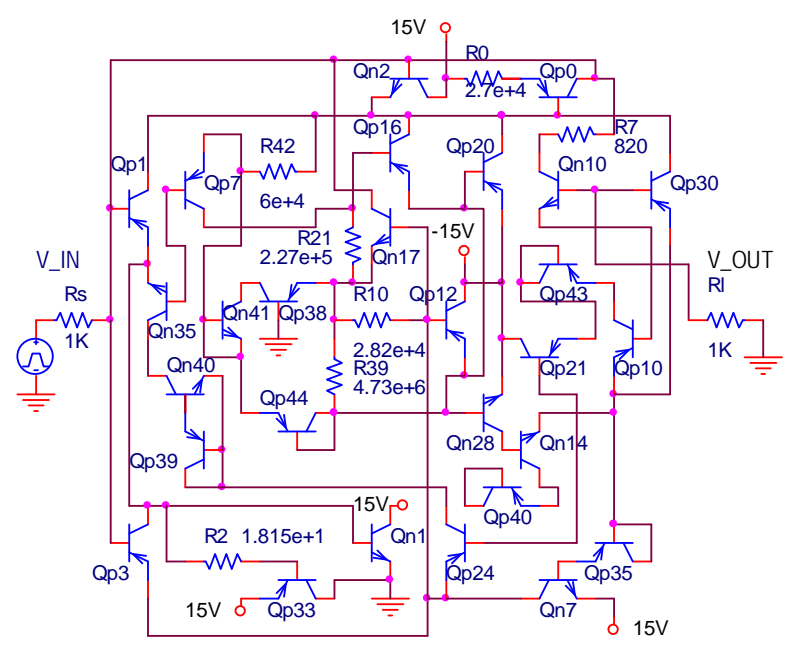

Figure 5. The evolved squaring circuit.

ponents with the fitness 0.0302 .

The best-of-run circuit (Figure 6) for the problem of designing a cube root circuit appeared at generation 152 and had 39 components with the fitness 0.2508 . The best-of-run circuit (Figure 7) for the problem of designing a cubing circuit appeared at generation 78 and has 44 components with the fitness 0.00614 . 


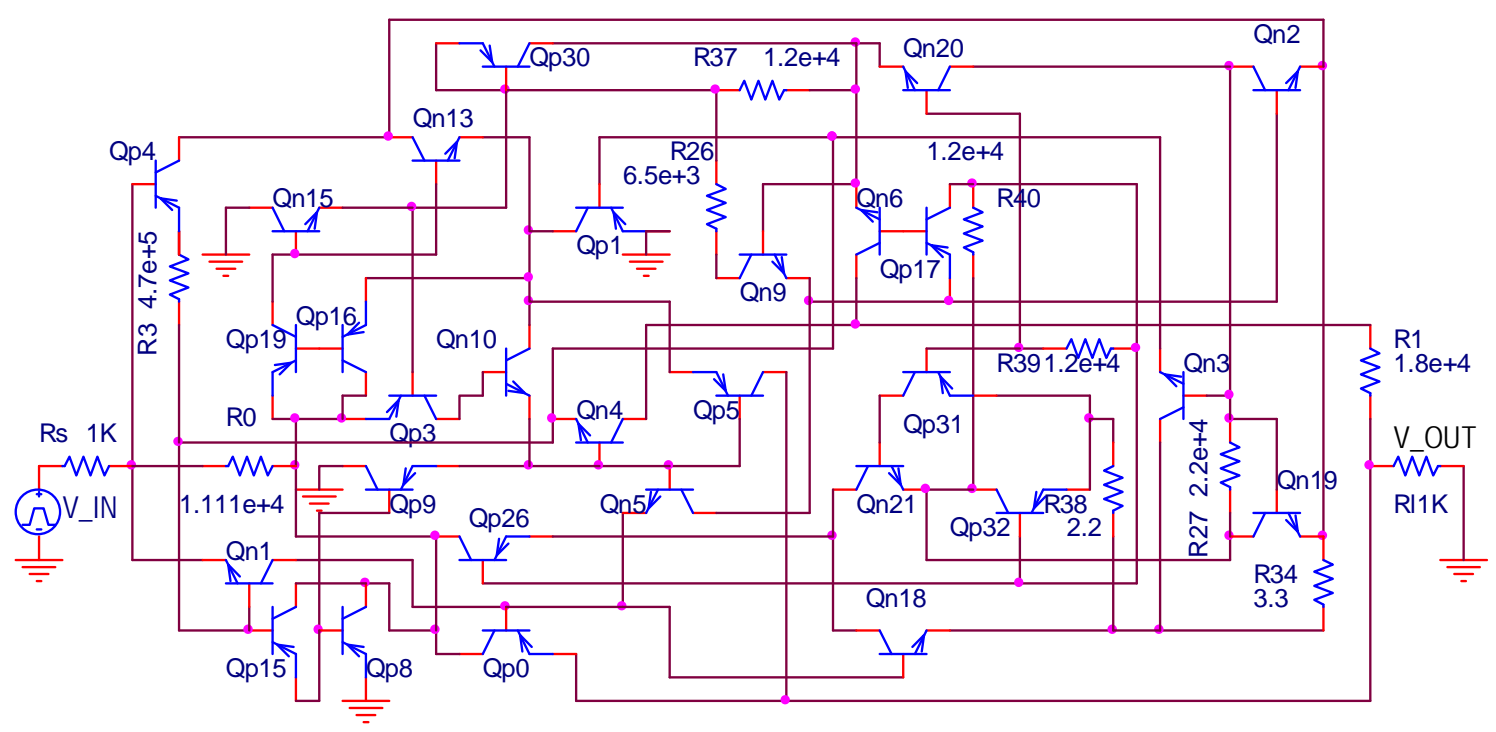

Figure 6. The evolved cube root circuit.

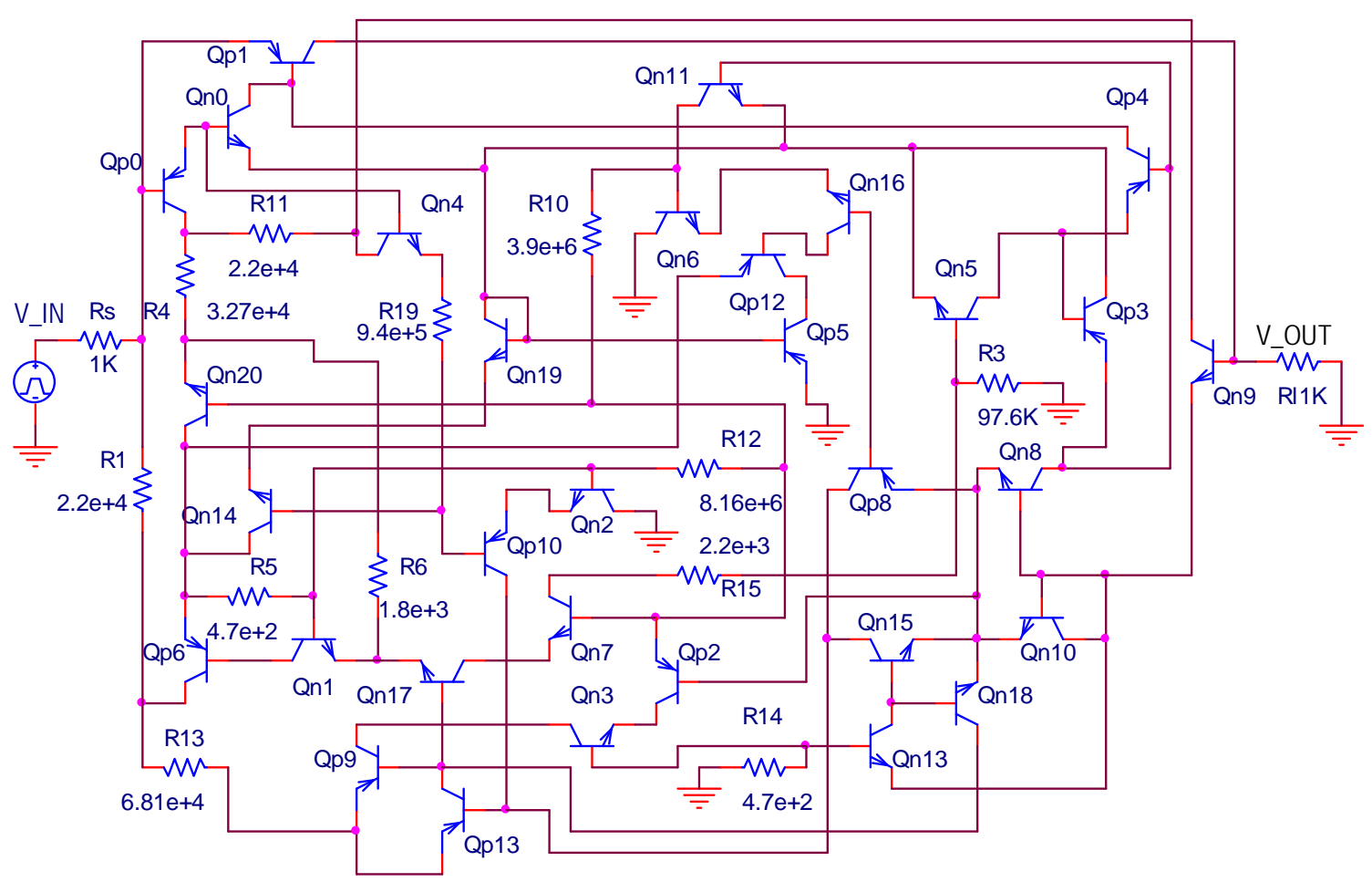

Figure 7. The evolved cubing circuit.

The schematics published in [3,9,20,25] enabled us to source-code them, analyze their netlists in PSPICE and get the fitness values appropriate for comparison. Both DC and transient analysis gave us identical results for each schematic, what, together with other published data, let us to aggregate all the data into Tables 3 and 4. For some circuits from [9] we got exactly the same fitness values, the last fact ensured us that we chose the right transistor models (SPICE default models as well as in $[3,20])$ and other simulation parameters. The most right column of the tables suggests the relative comparison between the value received in this paper and the best corresponding values from the past. As it could be noticed, by 15 from 16 comparable positions our results are 
Table 3. Comparison with circuits published before.

\begin{tabular}{|c|c|c|c|c|}
\hline Author & $\begin{array}{c}\text { Koza } \\
\text { et al. [9] }\end{array}$ & $\begin{array}{c}\text { Mydlowec } \\
\text { et al. [3] }\end{array}$ & This work & $\begin{array}{c}\text { Improve- } \\
\text { ment } \\
\text { (times) }\end{array}$ \\
\hline \multicolumn{5}{|c|}{ Square root } \\
\hline Average error, $\mathrm{mV}$ & 183.57 & 20.00 & 9.23 & 2.2 \\
\hline Fitness value & 3.855 & 70.403 & 0.194 & 18.9 \\
\hline Component No & 64 & 39 & 22 & 1.8 \\
\hline Evaluation No & Data n/a & $6,7 \mathrm{E}+9$ & $3,7 \mathrm{E}+6$ & 1800 \\
\hline \multicolumn{5}{|c|}{ Squiring } \\
\hline Average error, $\mathrm{mV}$ & Data $\mathrm{n} / \mathrm{a}$ & 27.00 & 1.44 & 18.7 \\
\hline Fitness value & Not converged & 4.812 & 0.0302 & 159.3 \\
\hline Component No & 39 & 37 & 35 & 1.1 \\
\hline Evaluation No & Data n/a & $1,1 \mathrm{E}+9$ & $2,7 \mathrm{E}+6$ & 407 \\
\hline \multicolumn{5}{|c|}{ Cube root } \\
\hline Average error, $\mathrm{mV}$ & 80.00 & - & 11.90 & 6.7 \\
\hline Fitness value & 1.68 & - & 0.2508 & 6.7 \\
\hline Component No & 50 & - & 39 & 1.3 \\
\hline Evaluation No & $3.8 \mathrm{E}+7$ & - & $4.5 \mathrm{E}+6$ & 8.4 \\
\hline
\end{tabular}

Table 4. Comparison of evolved cubing circuit with ones published previously.

\begin{tabular}{lccccc}
\hline \multicolumn{1}{c}{ Author } & $\begin{array}{c}\text { Koza } \\
\text { et al.[9] }\end{array}$ & $\begin{array}{c}\text { Streeter } \\
\text { et al.[19] }\end{array}$ & $\begin{array}{c}\text { Cipriani } \\
\text { et al. [25] }\end{array}$ & $\begin{array}{c}\text { This } \\
\text { work }\end{array}$ & $\begin{array}{c}\text { Improve- } \\
\text { ment } \\
\text { (times) }\end{array}$ \\
\hline Aveature & \multicolumn{5}{c}{ Cubing } \\
Fitness value & 0.0219 & Data n/a & Data n/a & 0.0061 & 3.6 \\
Component No & 56 & 47 & 12 & 44 & 0.3 \\
Evaluation No & Data n/a & $2.94 \mathrm{E}+6$ & - & $2.34 \mathrm{E}+6$ & 1.3 \\
\hline
\end{tabular}

considerably better. Notably, that the best by size (12 components) conventionally designed cubing circuit from [25] has an average error $(7.13 \mathrm{mV}) 25$ times larger than that one $(0.29 \mathrm{mV})$ of cubing circuit (44 components) evolved by us.

\section{Conclusions}

In this paper, we applied the unconstrained evolution towards the design of analogue computational circuits, on the example of cube root, cubing, square root and squiring functions. It was one of the first successful attempts of application of Evolutionary Strategy (ES) towards the large analog circuit synthesis. The technique presented is based on ES, oscillating length genotype, three types of mutation and substructure reuse. In general, the technique is plain with a simple algorithm. In all four cases, we successfully evolved circuits with fewer numbers of components at much less computer efforts with significantly better fitness.

The work discovered the capabilities of the technique developed by authors to find unconventional, economic and precise solutions. However, the designs presented are just simulated models, and are not guaranteed to be robust being in silicon. There are still "countervailing factors that impede progress toward industrial-strength automated design of analog circuits" [27] such as mismatching properties of devices, variation in the circuit's power supply and different operating temperatures.

Reaching the powerful evolutionary technique that capable of finding theoretical solutions was only the first stage of the research, whereas the second round is purposed to be strengthening the designs evolved towards the robust circuits in silicon.

\section{REFERENCES}

[1] A. Thompson, "Artificial Evolution in the Physical World," In: Gomi, Ed., Evolutionary Robotics, AAI Books, 1997, pp. 101-125.

[2] R. Ingo, "Optimierung Technischer Systeme Nach Prinzipien der Biologischen Evolution," $\mathrm{PhD}$ Dissertation, Technical University of Berlin, 1970.

[3] W. Mydlowec and J. Koza, "Use of Time-Domain Simulations in Automatic Synthesis of Computational Circuits Using Genetic Programming," Genetic and Evolutionary Computation Conference, Las Vegas, 2000, pp. 187-197.

[4] T. McConaghy and G. G. E. Gielen, "Globally Reliable Variation-Aware Sizing of Analog Integrated Circuits via ResponseSurfaces and Structural Homotopy," IEEE Transactions on Computer-Aided Design, Vol. 28, No. 11, November 2009, pp.1627-1640.

[5] K. Kim, A. Wong and H. Lipson, "Automated Synthesis of Resilient and Tamper-Evident Analog Circuits without a Single Point of Failure," Genetic Programming and Evolvable Machines (online), 2009.

[6] A. Thompson and P. Layzell, "Evolution of Robustness in an Electronics Design," International Conference on Intelligent Engineering Systems, Springer, 2000, pp. 218-228.

[7] A. Stoica, D. Keymeulen, T. Arslan, V. Duong, R. Zebulum, I. Ferguson and X. Guo, "Circuit Self-Recovery Experiments in Extreme Environments," Proceedings of the 2004 NASA/DoD Conference on Evolvable Hardware, Seattle, June 2004, pp. 142-145

[8] J. Walker, J. Hilder and A. Tyrrell, "Towards Evolving Industry-Feasible Intrinsic Variability Tolerant CMOS Designs," 11th IEEE Congress on Evolutionary Computation, Trondheim, May 2009, pp. 1591-1598.

[9] J. Koza, "Automated Synthesis of Computational Circuits Using Genetic Programming," IEEE Conference on Evolutionary Computation, Piscataway, 1997, pp. 447-452.

[10] R. Zebulum, M. Pacheco and M. Vellasco, "Comparison of Different Evolutionary Methodologies Applied to Electronic Filter Design," IEEE Conference on Evolutionary Computation, Piscataway, 1998, pp. 434-439.

[11] J. Lohn and S. Colombano, "Automated Analog Circuit Synthesis Using a Linear Representation," The 2nd International Conference on Evolvable Systems: From Bi- 
ology to Hardware, Springer-Verlag, 1998, pp. 125-133.

[12] C. Goh and Y. Li, "GA Automated Design and Synthesis of Analog Circuits with Practical Constraints," Proceedings of the 2001 Congress on Evolutionary Computation, Vol. 1, 2001, pp. 170-177.

[13] S. Ando and H. Iba, "Analog Circuit Design with a Variable Length Chromosome," Proceedings of the 2000 Congress on Evolutionary Computation, Vol. 2, 2000, pp. 994-1001.

[14] J. Grimbleby, "Hybrid Genetic Algorithms for Analogue Network Synthesis," Proceedings of the 1999 Congress on Evolutionary Computation, 1999, pp. 1781-1787.

[15] J. Hu, X. Zhong and E. Goodman, "Open-ended Robust Design of Analog Filters Using Genetic Programming," Genetic \& Evolutionary Computation Conference, Vol. 2, 2005, pp. 1619-1626.

[16] Y. Sapargaliyev and T. Kalganova, "On Comparison of Constrained and Unconstrained Evolutions in Analogue Electronics on the Example of LC Low-Pass Filters," IEICE Transactions on Electronics, Vol. E89-C, No. 12, December 2006, pp. 1920-1927.

[17] Z. Gan, Z. Yang, G. Li and M. Jiang, "Automatic Synthesis of Practical Passive Filters Using Clonal Selection Principle-Based Gene Expression Programming," Proceedings of the 7th International Conference on Evolvable Systems: From Biology to Hardware (ICES'07), Vol. 4684, 2007, pp. 1611-3349.

[18] S. Chang, H. Hou and Y. Su, "Automated Passive Filter Synthesis Using a Novel Tree Representation and Genetic Programming," IEEE Transactions on Evolutionary Computation, Vol. 10, No. 1, February 2006, pp. 93-100.

[19] D. Smith, “A Square Root Circuit to Linearize Feedback in Temperature Controllers," Journal of Physics E: Sci- entific Instruments, Vol. 5, No. 6, 1972, pp. 528-529.

[20] M. Streeter, M. Keane and J. Koza, "Iterative Refinement of Computational Circuits Using Genetic Programming," Proceeding of the 2002 Genetic and Evolutionary Computation Conference, 2002, pp 877-884.

[21] T. Dastidar, P. Chakrabarti and P. Ray, "A synthesis System for Analog Circuits Based on Evolutionary Search and Topological Reuse," IEEE Transactions on Evolutionary Computation, Vol. 9, No. 2, 2005, pp. 211-224.

[22] T. Sripramong and C. Toumazou, "The Invention of CMOS Amplifiers Using Genetic Programming and Current-Flow Analysis," IEEE Transactions on Computer-Aided Design of Integrated Circuits and Systems, Vol. 21, No. 11, 2002, pp.1237-1252.

[23] C. Mattiussi and D. Floreano, "Analog Genetic Encoding for the Evolution of Circuits and Networks," IEEE Transactions on Evolutionary Computation, Vol. 11, No. 5, 2007, pp. 596-607.

[24] A. Das and R. Vemuri, "An Automated Passive Analog Circuit Synthesis Framework using Genetic Algorithms," Proceedings of the IEEE Computer Society Annual Symposium on VLSI, 2007, pp. 145-152.

[25] S. Cipriani and A. Takeshian, "Compact Cubic Function Generator," U. S. Patent 6,160,427, Filed 4 September 1998, Issued December 12, 2000.

[26] OrCAD, Inc. "OrCad PSpice User's Guide," OrCAD, USA, 2004. http://www.electronics-lab.com/downloads/ schematic/013/tutorial/PSPCREF.pdf

[27] J. Koza, L. Jones, M. Keane, M. Streeter and S. Al-Sakran, "Toward Automated Design of Industrial-Strength Analog Circuits by Means of Genetic Programming," Genetic Programming Theory and Practice II, Kluwer Academic Publishers, Boston, 2004. 